INTERNATIONAL JOURNAL OF SCIENTIFIC RESEARCH

\title{
FEMORAL HERNIA MASQUERADING AS ACUTE GROIN PAIN: A DIAGNOSTIC AND SURGICAL CHALLENGE
}

\section{General Surgery}

\section{Swapnil Sen}

MBBS, DNB (General Surgery), FIAGES, Resident Surgeon, Department of General Surgery, Vivekananda Institute of Medical Sciences, West Bengal University of Health Sciences, Kolkata, West Bengal, India

\section{ABSTRACT}

Femoral hernia is a type of groin hernia, where the content protrudes through the femoral ring. Common contents in femoral hernias are, preperitoneal fat and small bowel, in some rare cases, sac containing an appendix, Meckel's diverticulum, ectopic testis, and stomach are reported.1 A femoral hernia presents as a mass or bulge below the inguinal ligament. Sometimes, femoral hernia presents as a bulge over the inguinal canal. In this case, the femoral hernia still exits inferior to the inguinal ligament through the femoral canal but ascends in a cephalad direction. 2 Sometimes, femoral hernias may present in an occult manner with unexplained pain in the groin.

We report a single case of Richter's type strangulated femoral hernia at a tertiary care apex institution of Eastern India. This case of femoral herniation presented atypically as groin pain without any swelling initially. This was followed by swelling with pain at the groin above the inguinal ligament after a few days.

\section{KEYWORDS}

Femoral hernia; Groin pain; Ileo-ileal anastomosis

\section{Case Report:}

A 56 year old lady was admitted with sudden onset severe pain in the right groin in a local medical facility. Local examination of the Right groin and the whole abdomen were normal. Imagings of the groin and Whole Abdomen with Ultrasound and blood parameters were normal. She was initially managed conservatively and discharged after 3 days. After a week, she again developed sudden onset pain in the right groin, and this time with a bulge at the same site. This was subsequently associated with non passage of flatus and faeces and multiple episodes of vomiting. She was then admitted at our hospital.

On clinical examination, she was alert, conscious, afebrile, dehydrated, pulse $110 / \mathrm{min}$, blood pressure was $120 / 70 \mathrm{~mm} \mathrm{Hg}$. On examination of both groins, left groin was normal. There was a swelling in the right groin approximately $3.5 \mathrm{~cm}$ in diameter just above the pubic tubercle. The local area was hyperaemic and exquisitely tender. There was no expansile cough impulse over the swelling and it was irreducible and nonpulsatile. On per abdominal examination, Abdomen was mildly distended with guarding in the right lower quadrant. Hyperperistaltic sounds were noted. A clinical diagnosis of obstructed right inguinal hernia was made.

The laboratory parameters on admission were Total leucocyte count$10800 / \mathrm{cu}$ mm with neutrophilic predominance, Serum Urea- $36 \mathrm{mg} / \mathrm{dl}$, Serum Creatinine- $0.9 \mathrm{mg} / \mathrm{dl}$, Albumin- $2.6 \mathrm{~g} / \mathrm{dl}$, coagulation profile was normal and X-Ray Chest was within normal limits. Ultrasound of right groin was suggestive of an obstructed right femoral hernia. XRay Abdomen revealed multiple air fluid levels suggestive of small bowel obstruction. Contrast Enhanced Computerized Tomography of Whole Abdomen revealed Right sided femoral Hernia with ileum as contents with narrow neck and distention of small bowel loops.

Patient was resuscitated with intravenous fluids, antibiotics, analgesics, nasogastric suction and per urethral catheterisation and was then planned for emergency surgery. Under general anesthesia, exploration of the right groin was performed. With the classic inguinal approach, incision was made on the skin and carried down through the subcutaneous layers; the external oblique aponeurosis was opened along with its fibers till the internal inguinal ring was exposed. Following this the floor of the inguinal canal (fascia transversalis) was opened, and the femoral hernia sac was exposed. After exploration of the mass, we confirmed the diagnosis of the femoral hernia. Sac was opened at the fundus. Toxic fluid was aspirated. Gangrenous loop of small bowel was found. The neck was released after dividing the Inguinal ligament near its medial attachment. Resection of the gangrenous loop of bowel approximately $10 \mathrm{~cm}$ was done and an end to end ileoileal anastomosis was done in single layer in an interrupted fashion. Following this, the sac was ligated at the level of peritoneal cavity. Round ligament of the right side was ligated and divided. No indirect or direct inguinal inguinal hernial sac was found. The posterior wall was repaired with interrupted absorbable sutures. Hernia repair was done using non absorbable sutures with inguinal ligament and iliopubic tract inferiorly, Cooper ligament posteriorly, femoral sheath laterally, with the iliopubic tract and lacunar ligament medially. External oblique was closed with absorbable sutures. A vacuum suction drain was placed in the subcutaneous layer. Skin was closed with non absorbable sutures.

Post operative period was uneventful. She received total parenteral nutrition postoperatively for 3 days. Nasogastric tube, catheter and drain were removed after 3 days. Stitches were removed after 7 days and the patient was discharged in a haemodynamically stable condition.

\section{DISCUSSION:}

An occult hernia is a hernia where physical examination fails to demonstrate a hernia mass or defect, but a hernia is identified on surgical exploration. ${ }^{3}$ An occult hernia can be completely asymptomatic or it may present with unexplained chronic groin or pelvic pain. ${ }^{4}$

Femoral hernias are challenging conditions, not only because their diagnosis is often missed, but also because if left undiagnosed and untreated, their complications are severe and often life-threatening.

Femoral hernias are elusive conditions that despite having lifethreatening complications are often undiagnosed in asymptomatic patients. ${ }^{6,7}$ They are less common than inguinal hernias and occur more frequently in females.

Despite the fact that femoral hernias account for only $2-4 \%$ of all groin hernias, their timely and correct diagnosis is vital due to the increased mortality associated with emergency surgery for their complications. This, however, is not always easy. Femoral hernias are commonly missed or misdiagnosed as less serious conditions, leaving surgeons to deal with their complications in the acute setting, where mortality has been found to be 10 fold. $^{6-8}$

\section{Figures:}

Figure 1:

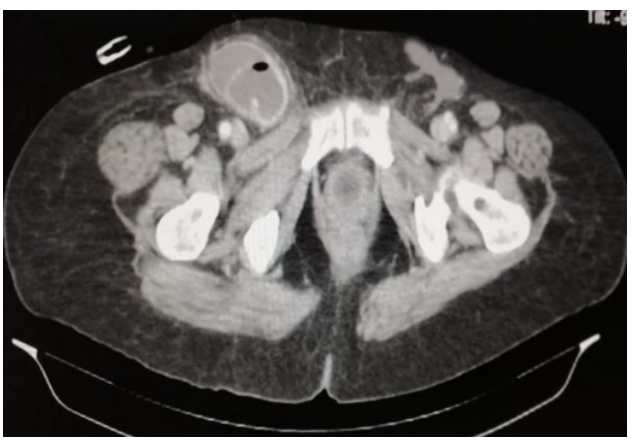




\section{Figure 2:}

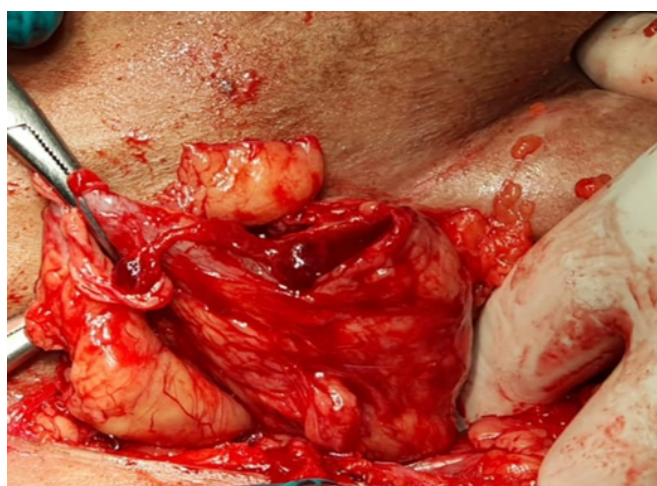

Figure 3:

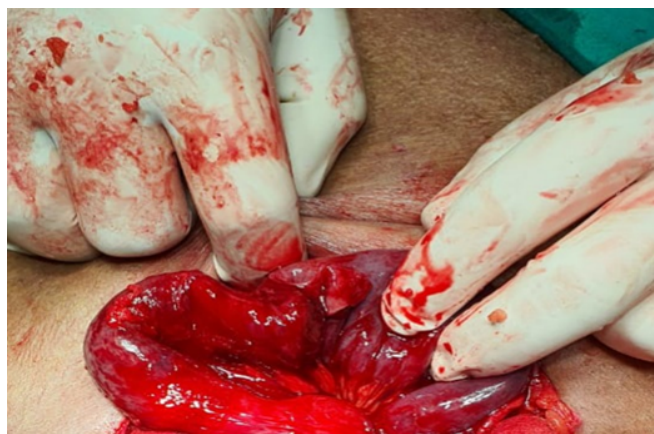

Figure 4:

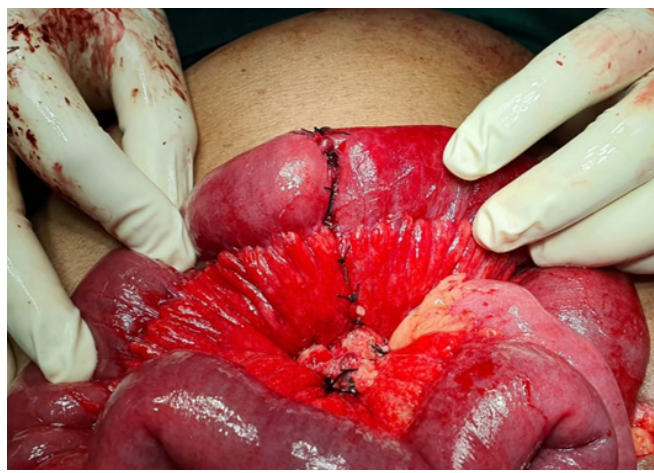

Legends:

Figure 1: Contrast Enhanced Computerized Tomography Scan of Whole Abdomen showing Right sided Femoral Hernia (axial cut) with ileal loop as contents

Figure 2: Intra operative image of Right sided Femoral Hernia Sac with narrow neck

Figure 3: Gangrenous loop of distal ileum

Figure 4: Intra operative image of completed Resection of part of gangrenous loop of bowel and Ileo-ileal anastomosis

\section{REFERENCES}

Marioni P. Metastatic carcinoma with small intestine in a femoral hernia, with a review of contents of femoral hernia sacs. Can Med Assoc J. 1960;82:1081-2.

2. Malangoni MA, Rosen MJ, Hernias. In: Townsend CM, Beauchamp RD, Evers BM, Mattox KL, editors. Sabiston Textbook Of Surgery: The Biological Basis Of Modern Surgical Practice. First South Asia Edition. New Delhi: RELX India Private Limited; 2016.Pp.1104.

3. Miller J, Cho J, Michael MJ, Saouaf R, Towfigh S. Role of Imaging in the diagnosis of occult hernias. JAMA Surg. 2014;149(10):1077-1080.

4. Matsevych OY, Koto MZ, and Becker JHR. Multiple concurrent bilateral groin hernias in a single patient; a case report and a review of uncommon groin hernias: A possible source of persistent pain after successful repair. Int J Surg Case Rep. 2016; 29: 204-207.

5. Arkoulis N, Savanis G, and Simatos G. Richter's type strangulated femoral hernia containing caecum and appendix masquerading as a groin abscess J Surg Case Rep. 2012 Jun; 2012(6): 6 .

6. Whalen HR, Kidd GA, O’Dwyer PJ. Femoral Hernias. BMJ 2011;343:d7668.

7. Naude GP, Ocon S, Bongard F. Femoral Hernia: The Dire Consequences of a Missed Diagnosis. Am J Emerg Med. 1997. Nov;15(7):680-2.

8. Dahlstrand U, Wollert S, Nordin P, Sandblom G, Gunnarsson U. Emergency femoral hernia repair. Astudy based on a national register. Ann Surg 2009;249:672-6. 